\title{
Determination of the UV cut-off from the observed value of the Universe acceleration.
}

\author{
S.L. Cherkas $\dagger$ and V.L. Kalashnikov \\ $\dagger$ Institute for Nuclear Problems, Bobruiskaya 11, Minsk 220050, Belarus \\ $\ddagger$ Technische Universität Wien, Gusshausstrasse 27/387, Vienna A-1040, Austria
}

\begin{abstract}
It is shown that use of the equation of motion of the Universe scale factor allows calculation of the contribution of the vacuum fluctuations to the acceleration of the Universe expansion. Renormalization of the equation is needed only in the case of massive particles. Under a known number of the different kinds of fundamental fields, this provides the determination of momentum of the ultraviolet cut-off from the observed value of acceleration.
\end{abstract}

PACS numbers: 95.36+x, 98.80.-k, 11.10.Gh 


\section{Introduction}

Discovery of the Universe accelerated expansion [1, 2] stirs up theoretical analysis aimed at explanation of the observational data. On the one hand, most of approaches are based on exploration of the new kinds of substance (quintessence, Chaplygin's gases, phantom field, ghost condensate, and so on) or of the new concepts of space-time (extradimensions, holographic principle, string multiple vacuum) [3]. On the other hand, it was proposed that such an explanation can be based on an assumption that Universe or its constituents are inhomogeneous [4, 5, 6]. The extreme point of view [4] is to consider Universe as inhomogeneous at the super-Hubble scales and to assume that we live at the edge of "Hubble-bubble" and thereby can observe either accelerated or decelerated expansion of Universe, which is not described by the Friedmann equations. Also, one can assume that the accelerated expansion is produced by an internal motion of matter on a smaller scale (like a galaxy formation one [6]) if the corresponding averaging procedure is defined for the Universe scale factor [5].

Nevertheless, it is still attractive to consider the QFT vacuum in a non-stationary space-time (i.e. in an expanding Universe) as the source of the Unverse acceleration. This can be achieved by exploring of two scales of Universe: $L$ and $1 / M_{p}$. Here $L$ is the "size" of Universe (of the order of its curvature or its inverse Hubble constant for a flat space-time), $M_{p}$ is the Planck mass associated with an appearance of new physics. Whereas the direct ("naive") consideration results in the huge value of vacuum density $\rho_{\text {vac }} \sim M_{p}^{4}$, the dimensional regularization of divergent expressions in a manner of Minkowski QFT gives the tiny value $\rho_{v a c} \sim L^{-4}$ [7, 8. In contrast to these approaches, there is a number of works [11, 12, 13, 14, 15, 16, 17, 18, 19] obtaining $\rho_{\text {vac }} \sim M_{p}^{2} L^{-2}$ that is consistent with the observed value of the Universe acceleration. Ref. [11] is simply an observation that the Schwarzschild radius $\rho_{v a c} L^{3} / M_{p}^{2}$ of Universe with the "mass" $\rho_{v a c} L^{3}$ has not to exceed the Universe "size" $L$. This excludes the "naive" value $\rho_{\text {vac }} \sim M_{p}^{4}$ and results in $\rho_{\text {vac }} \sim M_{p}^{2} L^{-2}$ as an upper limit. Other works are based on the Zeldovich's idea [20] assuming that the source of the Universe acceleration is not in itself the huge QFT vacuum energy but some another quantity, namely, the square root of dispersion of the vacuum energy fluctuations.

Although the direct UV cut-off on the Planck level in the Friedmann equation does not provide the observed value of the acceleration parameter (and is inconsistent with [11]), one can use it in the equation of motion of the Universe scale factor [21, 22]. It is found, that the effective cosmological constant results from the vacuum fluctuations of the massless scalar field and its value is concistent with $\rho_{v a c} \sim M_{p}^{2} L^{-2}$. The point is that, in contrast to the Friedmann equation which is held only up to some constant\& in

$\ddagger$ The works by L. Parker and collaborators 9,10 based on the nonperturbative approach stand apart. $\S$ Such a possibility in the quantum case was pointed out in Ref. 23. Also, arguments against a prevalence of the Friedmann equation were adduced in 24. In this work the quantization scheme for the equation of motion is proposed resulting in the quasi-Heisenberg operators which act in a space of solutions of the Wheeler-DeWitt equation. The hamiltonian constraint (i.e. the Friedmann equation) appears from two points of view there. The first one is properly the Wheeler-DeWitt equation and 
such an approach, the equation of motion contains difference of the potential and kinetic energies of the field oscillators. In Minkowski space-time this difference is exactly zero, because the virial theorem for an oscillator states that the kinetic energy is equal to the potential one in the virial equilibrium. However, in the expanding Universe the difference is proportional to the Hubble constant squared. This last tiny quantity is multiplied by the Planck-order momentum cut-off squared that gives true order of the acceleration parameter.

The aim of this work is to analyze a number of fundamental fields, which can contribute to the cosmological constant, and to investigate a possibility of exact determination of the UV cut-off from the observed value of the acceleration parameter.

\section{Contribution of the scalar particles}

In the case of the homogeneous isotropic flat Universe filled with the different species of matter, Lagrangian has the form [21]:

$$
\mathcal{L}=-\frac{M_{p}^{2} a^{\prime 2}}{2}+\mathcal{L}_{\text {scal }}+\mathcal{L}_{\text {ferm }}+\ldots
$$

where the metric $d s^{2}=a^{2}(\eta)\left(d \eta^{2}-d \boldsymbol{r}^{2}\right)$ is assumed and $M_{p}=\sqrt{\frac{3}{4 \pi G}}$ is the Planck mass. Matter Lagrangians are

$$
\begin{aligned}
\mathcal{L}_{\text {scal }} & =\frac{1}{2} \int\left(a^{2}\left(\partial_{\eta} \phi\right)^{2}-a^{2}(\boldsymbol{\nabla} \phi)^{2}-a^{4} m^{2} \phi^{2}\right) d^{3} \boldsymbol{r} \\
\mathcal{L}_{\text {ferm }} & =\int\left(\frac{i a^{3}}{2} \psi^{+} \partial_{\eta} \psi-\frac{i a^{3}}{2} \partial_{\eta} \psi^{+} \psi+i a^{3} \psi^{+} \boldsymbol{\alpha} \boldsymbol{\nabla} \psi-a^{4} m \psi^{+} \beta \psi\right) d^{3} \boldsymbol{r},
\end{aligned}
$$

where $\psi(\eta, \boldsymbol{r})$ is the spinor field and $\phi(\eta, \boldsymbol{r})$ is the scalar field and three dimensional volume of integration is assumed to be unity.

Let us turn to the Fourier-transform $\phi(\boldsymbol{r})=\sum_{\boldsymbol{k}} \phi_{\boldsymbol{k}} e^{i \boldsymbol{k} \boldsymbol{r}}$ that leads to the scalar field Lagrangian in the form

$$
\mathcal{L}_{\text {scal }}=\frac{1}{2} \sum_{\boldsymbol{k}} a^{2} \phi_{\boldsymbol{k}}^{\prime} \phi_{-\boldsymbol{k}}^{\prime}-a^{2} k^{2} \phi_{\boldsymbol{k}} \phi_{-\boldsymbol{k}}-a^{4} m^{2} \phi_{\boldsymbol{k}} \phi_{-\boldsymbol{k}}, .
$$

In the quasi-classical picture, where the Universe scale factor is considered classically whereas the scalar field is quantum, equations of motion are:

$$
\begin{aligned}
& \hat{\phi}_{\boldsymbol{k}}^{\prime \prime}+\left(k^{2}+a^{2} m^{2}\right) \hat{\phi}_{\boldsymbol{k}}+2 \frac{a^{\prime}}{a} \hat{\phi}_{\boldsymbol{k}}^{\prime}=0, \\
& M_{p}^{2} a^{\prime \prime}+\frac{1}{a} \sum_{\boldsymbol{k}} a^{2}<\hat{\phi}_{\boldsymbol{k}}^{\prime} \hat{\phi}_{-\boldsymbol{k}}^{\prime}>-a^{2} k^{2}<\hat{\phi}_{\boldsymbol{k}} \hat{\phi}_{-\boldsymbol{k}}> \\
& -2 a^{4} m^{2}<\hat{\phi}_{\boldsymbol{k}} \hat{\phi}_{-\boldsymbol{k}}>=0 .
\end{aligned}
$$

the second one is the relation for the quasi-Heisenberg operators. In the second sense, the constraint (Friedmann equation) is broken during the Universe evolution, when the potential energy of the scalar field begins to play a role. 
Here $<>$ denotes averaging over the vacuum state.

Quantization of the scalar field [7]

$$
\hat{\phi}_{\boldsymbol{k}}=\hat{\mathrm{a}}_{-\boldsymbol{k}}^{+} \chi_{k}^{*}(\eta)+\hat{\mathrm{a}}_{\boldsymbol{k}} \chi_{k}(\eta)
$$

leads to the operators of creation and annihilation with the commutation rules $\left[\hat{a}_{\boldsymbol{k}}, \hat{\mathrm{a}}_{\boldsymbol{k}}^{+}\right]=$ 1. The complex functions $\chi_{k}(\eta)$ are $\chi_{k}=\frac{1}{a \sqrt{2 k}} e^{-i k \eta}$ for $a=$ const.

In the general case they satisfy the relations [7]:

$$
\begin{aligned}
& \chi_{k}^{\prime \prime}+k^{2} \chi_{k}+2 \frac{a^{\prime}}{a} \chi_{k}^{\prime}=0, \\
& a^{2}(\eta)\left(\chi_{k} \chi_{k}^{\prime *}-\chi_{k}^{*} \chi_{k}^{\prime}\right)=i .
\end{aligned}
$$

Eqs. (8) admit the formal WKB solution [7]

$$
\chi_{k}=\frac{\operatorname{Exp}\left(-i \int_{0}^{\eta} W_{k}(\tau) d \tau\right)}{\sqrt{2} a(\eta) \sqrt{W_{k}(\eta)}},
$$

where function $W_{k}(\eta)$ satisfies the equation

$$
W_{k}^{\prime \prime}-\frac{3 W_{k}^{\prime 2}}{2 W_{k}}-2\left(k^{2}+m^{2} a^{2}-\frac{a^{\prime \prime}}{a}\right) W_{k}+2 W_{k}^{3}=0 .
$$

Adiabatic approximation consists in setting $W_{k}(\eta) \approx \sqrt{k^{2}+m^{2} a(\eta)^{2}-a^{\prime \prime}(\eta) / a(\eta)}$.

At first, we shall consider the massless case $m=0$ in which the equation of motion contains exact difference of the kinetic and potential energies of the field oscillators. This difference is zero in the vacuum state of Minkowski space. In the expanding Universe it takes the form

$$
\begin{aligned}
& a^{2}<\hat{\phi}_{\boldsymbol{k}}^{\prime} \hat{\phi}_{-\boldsymbol{k}}^{\prime}>-k^{2} a^{2}<\hat{\phi}_{\boldsymbol{k}} \hat{\phi}_{-\boldsymbol{k}}>=a^{2}\left(\psi_{k}^{\prime *} \psi_{k}^{\prime}-k^{2} \psi_{k}^{*} \psi_{k}\right) \\
& \approx \frac{1}{2 k}\left(-\frac{a^{\prime \prime}}{a}+\frac{a^{\prime 2}}{a^{2}}\right)+O\left(a^{\prime 3}\right)+O\left(a^{\prime} a^{\prime \prime}\right)+O\left(a^{\prime \prime \prime}\right),
\end{aligned}
$$

when the adiabatic approximation for the function $\psi_{k}$ is used. Using (11) in (6) yields the equation of motion for the Universe scale factor in the form

$$
M_{p}^{2} a^{\prime \prime}+\frac{1}{2 a}\left(-\frac{a^{\prime \prime}}{a}+\frac{a^{\prime 2}}{a^{2}}\right) \sum_{k} \frac{1}{k}=0 .
$$

Coming from the summation over $\boldsymbol{k}$ (in the general case it has to include summation over particle species) to the integration, we have

$$
\sum_{\boldsymbol{k}} \frac{1}{k}=\frac{4 \pi}{(2 \pi)^{3}} \int_{0}^{k_{\max }} k d k=\frac{k_{\max }^{2}}{4 \pi^{2}}=\kappa_{\max }^{2} \frac{a_{0}^{2}}{4 \pi^{2}},
$$

where $\kappa_{\max }=\frac{k_{\max }}{a_{0}}$ is the cutting parameter of the physical momentums and $a_{0}=a(0)$ is the present day $(\eta=0)$ scale factor. Finally, we come to the equation

$$
M_{p}^{2} a^{\prime \prime}=\rho a^{3}-\frac{1}{2 a}\left(-\frac{a^{\prime \prime}}{a}+\frac{a^{\prime 2}}{a^{2}}\right) N_{s c} \kappa_{\max }^{2} \frac{a_{0}^{2}}{4 \pi^{2}},
$$


where the density of classical matter (dust) $\rho=\frac{\rho_{0} a_{0}^{3}}{a^{3}}=\frac{\Omega_{m} M_{p}^{2} \mathcal{H}^{2} a_{0}}{2 a^{3}}$ and the number $N_{s c}$ of species of scalar particles are introduced. Here $\Omega_{m}=\rho_{0} / \rho_{\text {crit }}$ should be read as denoting the matter relative density and $\mathcal{H}=\left.\frac{a^{\prime}}{a}\right|_{\eta=0}$.

Consideration of Eq. (14) at $\eta=0$ allows determining the UV cut-off $\kappa_{\max }$ of physical momentum from the observed present day value of the Universe acceleration

$$
\kappa_{\max }=\left.\frac{2 \pi M_{p}}{\sqrt{N_{s c}}} \sqrt{\frac{2 a^{\prime \prime} a / a^{\prime 2}-\Omega_{m}}{a^{\prime \prime} a / a^{\prime 2}-1}}\right|_{\eta=0} .
$$

Now let us return to the massive particles. In this case there is no an exact difference of the kinetic and potential energies in the equation of motion because the multiplier 2 appears in Eq. (6) . Subtraction of the main terms corresponding to the Minkowski space-time can be used in this case as a primitive renormalization. Let us remind that for $a=$ const

$$
\begin{aligned}
& a^{4}<\phi_{k}^{\prime} \phi_{-k}^{\prime}>=<\pi_{k} \pi_{-k}>=\frac{1}{2} a^{2} \sqrt{k^{2}+m^{2} a^{2}}, \\
& <\phi_{k} \phi_{-k}>=\frac{1}{2 a^{2} \sqrt{k^{2}+m^{2} a^{2}}} .
\end{aligned}
$$

The renormalization consists in rewriting of the equation of motion in the form

$$
a^{\prime \prime} M_{p}^{2}-\rho a^{3}=-\sum_{k} \frac{\theta_{k}}{a^{3}}-a\left(k^{2}+2 a^{2} m^{2}\right) \chi_{k}
$$

where

$$
\begin{aligned}
& \theta_{k}=<\pi_{k} \pi_{-k}>-\frac{1}{2} a^{2} \sqrt{k^{2}+m^{2} a^{2}}, \\
& \chi_{k}=<\phi_{k} \phi_{-k}>-\frac{1}{2 a^{2} \sqrt{k^{2}+m^{2} a^{2}}} .
\end{aligned}
$$

The simplest way of further consideraton is to assume some particular dependence $a(\eta)$ and to calculate the quantity $\sum_{k} \frac{\theta_{k}}{a^{3}}-a\left(k^{2}+2 a^{2} m^{2}\right) \chi_{k}$. As it was done in [21], one can choose the dependence

$$
a(\eta)=a_{0}(1-\mathcal{H} \eta / 2)^{-2},
$$

which can be roughly associated with our present Universe because it gives the acceleration parameter $a^{\prime \prime} a / a^{\prime 2}=3 / 2$ and $a^{\prime} /\left.a\right|_{\eta=0}=\mathcal{H}$ (here $\mathcal{H}=H a_{0}$ and $H$ is the Hubble constant).

Calculation of the scalar field contribution to the Universe acceleration up to second order in $\mathcal{H}$ gives

$$
\begin{aligned}
& a^{\prime \prime} M_{p}^{2}-\rho a^{3}=-\sum_{k} \frac{\theta_{k}}{a^{3}}-a\left(k^{2}+2 a^{2} m^{2}\right) \chi_{k} \\
& \approx \sum_{k} \frac{\mathcal{H}^{2} k^{4}}{4 a_{0}\left(k^{2}+m^{2} a_{0}^{2}\right)^{5 / 2}}+\frac{3 a_{0} \mathcal{H}^{2} m^{2} k^{2}}{8\left(k^{2}+m^{2} a_{0}^{2}\right)^{5 / 2}}+O\left(\mathcal{H}^{4}\right) \\
& \approx \sum_{\boldsymbol{k}} \frac{\mathcal{H}^{2}}{4 a_{0} k}-\frac{a_{0} \mathcal{H}^{2} m^{2}}{4 k^{3}}+O\left(m^{4}\right)+O\left(\mathcal{H}^{4}\right)
\end{aligned}
$$




$$
\approx \frac{a_{0} \mathcal{H}^{2} \kappa_{\max }^{2}}{16 \pi^{2}}\left(1-\frac{2 m^{2}}{\kappa_{\max }^{2}} \ln \frac{\kappa_{\max }}{\kappa_{\min }}\right)
$$

where replacement of summation by integration over the momentum $k$ is proceeded and $\kappa_{\text {min }} \sim m$. One can see, that the mass terms are suppressed by the multiplier $m / \kappa_{\max } \sim m / M_{p}$ and are negligible, if one assumes that the mass of a particle is mach smaller than the Planck mass. Thus, one can conclude that the mass of the scalar particles has no impact.

\section{Fermions}

To consider the fermions in an expanding Universe, we keep to the earlier work [25] and the works [26, 27], where preheating of Universe was considered (see also [28, 29]).

After decomposing in the complete set of modes $\psi(\boldsymbol{r})=\sum_{\boldsymbol{k}} \psi_{\boldsymbol{k}} e^{i \boldsymbol{k} \boldsymbol{r}}$, the fermionic Lagrangian (3) takes the form

$$
\mathcal{L}_{f e r m}=\sum_{\boldsymbol{k}} \frac{i a^{3}}{2} \psi_{\boldsymbol{k}}^{+} \partial_{\eta} \psi_{\boldsymbol{k}}-\frac{i a^{3}}{2} \partial_{\eta} \psi_{\boldsymbol{k}}^{+} \psi_{\boldsymbol{k}}-a^{3} \psi_{\boldsymbol{k}}^{+}(\boldsymbol{\alpha} \boldsymbol{k}) \psi_{\boldsymbol{k}}-a^{4} m \psi_{\boldsymbol{k}}^{+} \beta \psi_{\boldsymbol{k}}
$$

The equations of motion are

$$
\begin{aligned}
& i \hat{\psi}_{k}^{\prime}-(\boldsymbol{\alpha} \boldsymbol{k}) \hat{\psi}_{\boldsymbol{k}}+i \frac{3 a^{\prime}}{2 a} \hat{\psi}_{\boldsymbol{k}}-m a \beta \hat{\psi}_{\boldsymbol{k}}=0, \\
& m_{p}^{2} a^{\prime \prime}+\sum_{\boldsymbol{k}} \frac{3}{2} a^{2}<i \hat{\psi}_{\boldsymbol{k}}^{+} \hat{\psi}_{\boldsymbol{k}}^{\prime}-i \hat{\psi}_{\boldsymbol{k}}^{\prime+} \hat{\psi}_{\boldsymbol{k}}-2 \hat{\psi}_{\boldsymbol{k}}^{+}(\boldsymbol{\alpha} \boldsymbol{k}) \hat{\psi}_{\boldsymbol{k}}> \\
& -4 a^{3} m<\hat{\psi}_{\boldsymbol{k}}^{+} \beta \hat{\psi}_{\boldsymbol{k}}>+\ldots=0,
\end{aligned}
$$

where quasi-classics is implied and dots denote matter (dust), scalar field, photon field and other terms.

Fermion field is quantized as

$$
\hat{\psi}_{\boldsymbol{k}}=\hat{b}_{-\boldsymbol{k}, s}^{+} v_{-\boldsymbol{k}, s}+\hat{a}_{\boldsymbol{k}, s} u_{\boldsymbol{k}, s},
$$

where the bispinor is $\|$ :

$$
\begin{aligned}
& u_{\boldsymbol{k}, s}(\eta)=\frac{i \chi_{k}^{\prime}+m a \chi_{k}}{a^{3 / 2}}\left(\begin{array}{c}
\varphi_{s} \\
\frac{\chi_{k}(\boldsymbol{\sigma k})}{i \chi_{k}^{\prime}+m \chi_{k} a} \varphi_{s}
\end{array}\right), \\
& \varphi_{+}=\left(\begin{array}{l}
1 \\
0
\end{array}\right) \text { and } \varphi_{-}=\left(\begin{array}{l}
0 \\
1
\end{array}\right) .
\end{aligned}
$$

The bispinor $v_{\boldsymbol{k}, s}$ is expressed as $v_{\boldsymbol{k}, s}=i \gamma^{0} \gamma^{2}\left(\bar{u}_{\boldsymbol{k}, s}\right)^{T}$, where the symbol $T$ denotes the transpose vector and $\bar{u}=u^{+} \gamma^{0}$. The functions $\chi_{k}(\eta)$ satisfy the equations

$$
\begin{aligned}
& \chi_{k}^{\prime \prime}+\left(k^{2}+m^{2} a^{2}-i m a^{\prime}\right) \chi_{k}=0, \\
& k^{2} \chi_{k} \chi_{k}^{*}+\left(a m \chi_{k}^{*}-i \chi_{k}^{\prime *}\right)\left(a m \chi_{k}+i \chi_{k}^{\prime}\right)=1 .
\end{aligned}
$$

I| Representation of the Dirac matrices is the same as in Refs. 30, 31. 
Eq. (23) reflects the time-dependence dictated by the bispinor equation of motion. Eq. (24) is required to satisfy the anti-commutator relation $\left\{\psi_{\boldsymbol{k}, s}^{+j}, \psi_{\boldsymbol{q}, \sigma}^{l}\right\}_{+}=\delta_{j l} \delta_{\boldsymbol{k q}} \delta_{s \sigma}$, so that the operators $a_{\boldsymbol{k}, s}$ satisfy $\left\{a_{\boldsymbol{k}, s} a_{\boldsymbol{q}, \sigma}^{+}\right\}_{+}=\delta_{\boldsymbol{k} \boldsymbol{q}} \delta_{s \sigma}$ and the similar relation is valid for $b_{\boldsymbol{k}, s}$. Also, one has to note that the left hand side of Eq. (24) is the integral of motion of Eq. (23).

Eq. (23) admits the WKB solution:

$$
\chi_{k}(\eta)=\frac{\exp \left(-i \int_{0}^{\eta} W_{k}(\tau) d \tau-\frac{1}{2} \int_{0}^{\eta} \frac{m a^{\prime}(\tau)}{W_{k}(\tau)} d \tau\right)}{\sqrt{2}\left(m a_{0}+\sqrt{k^{2}+m^{2} a_{0}^{2}}\right)^{1 / 2} \sqrt{W_{k}(\eta)}},
$$

where the function $W_{k}(\eta)$ satisfies the equation:

$$
W_{k}^{\prime \prime}-\frac{1}{2 W_{k}}\left(m^{2} a^{\prime 2}+3 W_{k}^{\prime 2}+4 m a^{\prime} W_{k}^{\prime}\right)+2 W_{k}^{3}-2\left(k^{2}+m^{2} a^{2}\right) W_{k}+m a^{\prime \prime}=0 .
$$

It should be noted, that Eq. (26) gives $W_{k}=k$ at $m=0$ and this can be seen directly from Eqs. (23) , (24) giving $\chi_{k}(\eta)=\frac{1}{\sqrt{2} k} e^{-i k \eta}$. In this case, the elementary calculations lead to

$$
\begin{aligned}
& \frac{3}{2} a^{2}<i \hat{\psi}_{\boldsymbol{k}}^{+} \hat{\psi}_{\boldsymbol{k}}^{\prime}-i \hat{\psi}_{\boldsymbol{k}}^{\prime+} \hat{\psi}_{\boldsymbol{k}}-2 \hat{\psi}_{\boldsymbol{k}}^{+}(\boldsymbol{\alpha} \boldsymbol{k}) \hat{\psi}_{\boldsymbol{k}}> \\
& =\frac{3}{a}\left(i \chi_{k}^{*} \chi_{k}^{\prime} k^{2}-i \chi_{k} \chi_{k}^{* \prime} k^{2}-i \chi_{k}^{* \prime} \chi_{k}^{\prime \prime}+i \chi_{k}^{\prime} \chi_{k}^{* \prime \prime}\right)=0 .
\end{aligned}
$$

Thus, according to (21) the massless fermions do not contribute to the Universe acceleration.

To analyze the massive fermions, let us assume the particular dependence $a(\eta)$ given by (18) and calculate the quantity

$$
\begin{aligned}
& \sum_{\boldsymbol{k}} \frac{3}{2} a^{2}<i \hat{\psi}_{\boldsymbol{k}}^{+} \hat{\psi}_{\boldsymbol{k}}^{\prime}-i \hat{\psi}_{\boldsymbol{k}}^{\prime+} \hat{\psi}_{\boldsymbol{k}}-2 \hat{\psi}_{\boldsymbol{k}}^{+}(\boldsymbol{\alpha} \boldsymbol{k}) \hat{\psi}_{\boldsymbol{k}}>-4 a^{3} m<\hat{\psi}_{\boldsymbol{k}}^{+} \beta \hat{\psi}_{\boldsymbol{k}}> \\
= & \sum_{\boldsymbol{k}} \frac{2 a_{0} m^{2}}{k}-\frac{3 a_{0} m^{2} \mathcal{H}^{2}}{k^{3}}-\frac{9 m \mathcal{H}^{2}}{4 k^{2}}+O\left(m^{3}\right)+O\left(\mathcal{H}^{4}\right) \\
\approx & \frac{a_{0}^{3} \kappa_{\max }^{2} m^{2}}{2 \pi^{2}}-\frac{9 a_{0} \mathcal{H}^{2} \kappa_{\max }^{2}}{8 \pi^{2}}\left(\frac{m}{\kappa_{\max }}+\frac{4 m^{2}}{3 \kappa_{\text {max }}^{2}} \ln \frac{\kappa_{\text {max }}}{\kappa_{\text {min }}}\right)
\end{aligned}
$$

contained in Eq. (21). One can see that after subtraction of the first term (which is equivalent to the "renormalization" (17) in the case of the scalar field), the remaining terms are suppressed by the multiplier $m / \kappa_{\max }$ in comparison with the acceleration term created by the vacuum of the massless scalar field.

It seems that the negligible contribution of the massive terms is a general feature. Indeed, the quantity $M_{p}^{2} a^{\prime \prime} / a$ is of the forth-order in mass. From the dimension arguments, it can be proportional to $\mathcal{H}^{2} \kappa_{\text {max }}^{2}, \mathcal{H}^{2} \kappa_{\text {max }} m, \mathcal{H}^{2} m^{2}$ and two last terms are negligible in comparison with the first one at $\kappa_{\max } \sim M_{p}$. Note, that the term $\mathcal{H} m \kappa_{\text {max }}^{2}$ is forbidden by the time-reversal invariance arising from the fluctuation origin (i.e. the vacuum fluctuations) of the quantity under consideration. 


\section{Conformally coupled scalar fields, photons and any conformal fields}

As one can see, that the massless fermions do not contribute to the Universe acceleration. The deep-laid reason (see for instance [32]) is conformality of the fermionic Lagrangian if $m=0$. Other conformal Lagrangians correspond to both photonic and scalar fields with the $R / 6$ term for the latter [7]. For shortness it is sufficiently to consider only the last Lagrangian, which takes the form:

$$
\mathcal{L}_{\text {conf }}=-\frac{1}{2} M_{p}^{2} a^{\prime 2}+\frac{1}{2} \sum_{\boldsymbol{k}}\left\{y_{\boldsymbol{k}}^{\prime} y_{-\boldsymbol{k}}^{\prime}-m^{2} a^{2} y_{\boldsymbol{k}} y_{-\boldsymbol{k}}-k^{2} y_{\boldsymbol{k}} y_{-\boldsymbol{k}}\right\},
$$

where $y_{\boldsymbol{k}}=a(\eta) \phi_{\boldsymbol{k}}$. One can see, that the field oscillators are uncoupled from the Universe scale factor in the case of $m=0$ and, thereby, do not contribute to the equation of motion for it. Equivalent conclusion, that the massless conformal scalar field does not produce a vacuum polarization has been done in [33].

\section{Gravitons}

Let us turn to the pure gravity. Small oscillations of the graviton oscillators near an equilibrium point can be considered on the basis of the perturbation theory [34]. It is known that the scalar and vector modes cannot produce free oscillations without the matter fields [34]. On the other hand, it is possible to expect that the matter fields are uncoupled from the scalar and vector perturbations at the Planck frequencies, that is the frequencies providing main contribution to the vacuum energy within the framework of our approach.

Thus, the remaining perturbations are the tensor ones existing in an empty space. Let us represent metric in the form

$$
g_{\mu \nu}=\gamma_{\mu \beta}\left(\delta_{\nu}^{\beta}+h_{\nu}^{\beta}+\frac{1}{2} h_{\rho}^{\beta} h_{\nu}^{\rho}\right)
$$

where $\gamma_{\mu \nu}=a^{2}(\eta) \operatorname{diag}\{1,-1,-1,-1\}$. All components of the perturbation $h_{\nu}^{\mu}(\eta, \boldsymbol{r})$ containing at least one zero index are equal to zero and the spatial components are represented as

$$
h_{n}^{j}(\eta, \boldsymbol{r})=\frac{2 \sqrt{6}}{M_{p}} \sum_{\boldsymbol{k}}\left(\left[\Phi_{\boldsymbol{k}}\right]_{n}^{j} \phi_{\boldsymbol{k}}(\eta)+\left[\mathcal{Y}_{\boldsymbol{k}}\right]_{n}^{j} y_{\boldsymbol{k}}(\eta)\right) e^{i \boldsymbol{k} \boldsymbol{r}} .
$$

The symmetric tensors $\Phi_{\boldsymbol{k}}$ and $\mathcal{Y}_{\boldsymbol{k}}$ describing two possible polarizations have the following properties: $\operatorname{Tr}\left[\Phi_{\boldsymbol{k}}\right]=\operatorname{Tr}\left[\mathcal{Y}_{\boldsymbol{k}}\right]=0, \quad \operatorname{Tr}\left[\mathcal{Y}_{\boldsymbol{k}} \mathcal{Y}_{-\boldsymbol{k}}\right]=\operatorname{Tr}\left[\Phi_{\boldsymbol{k}} \Phi_{-\boldsymbol{k}}\right]=1$, $\Phi_{\boldsymbol{k}} \cdot \boldsymbol{k}=\mathcal{Y}_{\boldsymbol{k}} \cdot \boldsymbol{k}=0$. For instance, when the $\boldsymbol{k}$-vector is directed along $x$-axis, these tensors look as:

$$
\mathcal{Y}_{\boldsymbol{k}}=\frac{1}{\sqrt{2}}\left(\begin{array}{ccc}
0 & 0 & 0 \\
0 & 1 & 0 \\
0 & 0 & -1
\end{array}\right), \quad \Phi_{\boldsymbol{k}}=\frac{1}{\sqrt{2}}\left(\begin{array}{ccc}
0 & 0 & 0 \\
0 & 0 & 1 \\
0 & 1 & 0
\end{array}\right)
$$

I We are going to do calculations up to the second-order in $h$, thus the quadratic terms on $h$ are kept in the metric. 
For an arbitrary direction, they can be obtained from these expressions through the $\mathrm{SO}(3)$-transformation.

The next step is to substitute Eqs. (30), (31) in the gravity action $S=$ $-\frac{M_{p}^{2}}{12} \int \mathcal{G} \sqrt{-g} d^{4} x$, where $\mathcal{G}=g^{\alpha \beta}\left(\Gamma_{\alpha \nu}^{\rho} \Gamma_{\beta \rho}^{\nu}-\Gamma_{\alpha \beta}^{\nu} \Gamma_{\nu \rho}^{\rho}\right)$ according to [35]. Finally, in the second-order in $y_{\boldsymbol{k}}, \phi_{\boldsymbol{k}}$ we come to

$S=\int\left(-\frac{M_{p}^{2} a^{\prime 2}}{2}+\frac{1}{2} \sum_{\boldsymbol{k}} a^{2} \phi_{\boldsymbol{k}}^{\prime} \phi_{-\boldsymbol{k}}^{\prime}-a^{2} k^{2} \phi_{\boldsymbol{k}} \phi_{-\boldsymbol{k}}+a^{2} y_{\boldsymbol{k}}^{\prime} y_{-\boldsymbol{k}}^{\prime}-a^{2} k^{2} y_{\boldsymbol{k}} y_{-\boldsymbol{k}}\right) d \eta$.

Thus the tensor wave contributes to the vacuum energy as two scalar fields.

\section{Conclusion}

In the case of the massless particles and when the conformal time is used, it is shown that the equation of motion of the Universe scale factor contains the difference of potential and kinetic energies of field oscillators. Calculation of the mean value of this quantity over the vacuum state leads to the Universe accelerated expansion. The observed value of the Universe acceleration allows determining the UV cut-off of the physical momentum given by Eq. (15), which contains the dust density and the number of species of scalar fields $N_{s c}$.

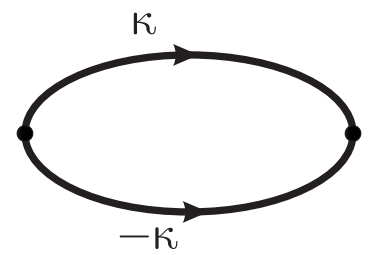

Figure 1. Diagram of vacuum polarization. Bold lines denote a virtual particle propagation in a background metric of expanding Universe.

Let us make some notes about the number of species of scalar particles. At first sight, there appear to exists a number of the scalar particles like pions. However, the momentum $\kappa$ of a virtual particle appears in the loop diagram and the main contribution arises from $\kappa$ of the Planck mass order. Under the circumstances, the composite particles decompose into their constituents. Thus, $N_{s c}$ should be read as a number of the fundamental scalar fields.

In a minimal variant of the standard model there is the $\mathrm{SU}(2)$ duplet of complex scalar fields [36], i.e. four scalar degrees of freedom. Adding two degrees of freedom of the tensor gravitational wave, one comes to $N_{c s}=6$ and the substitutions of $a^{\prime \prime} a / a^{\prime 2}=3 / 2$ and $\Omega_{m}=1 / 3$ in Eq. (15) lead to the UV cut-off of the physical momentum: $\kappa_{\max }=\frac{4 \sqrt{2} \pi M_{p}}{3}=2 \sqrt{\frac{2 \pi}{3 G}}$.

Still, the scale of the Grand Unfed Theory $10^{15}-10^{16} \mathrm{GeV}$ seems closer to the Planck scale and, hence there are ten scalar degrees of freedom for SU(5) (i.e. total twelve). Then $\kappa_{\max }=2 \sqrt{\frac{\pi}{3 G}}$. 
Scalar sectors of both the string theory and the supersymmetry one providing a number of the scalar superpartners of fermions demand a deeper insight.

Let us remind that photons, fermions and conformally coupled scalars do not contribute to the Universe acceleration. The masses of all particles gives a negligible contribution after separation of the term corresponding to the Minkowski space.

\section{References}

[1] Perlmutter S et al 1999 Astrophys. J 517565

[2] Riess A et al 1998 Astron. J 1161009

[3] Copeland E J, Sami M and Tsujikawa S Preprint hep-th/0603057

[4] Kolb E W, Matarrese S, Notari A, and Riotto A 2005 Phys. Rev. D 71023524

[5] Buchert T, Larena J and Alimi J-M 2006 Class.Quant.Grav. 236379

[6] Räsänen S Preprint astro-ph/0607626

[7] Birrell N D and Davis P C W 1982 Quantum Fields in Curved Space (Cambridge, Univ. Press)

[8] Onemli V K, Woodard R P 2004 Phys.Rev. D 70107301

[9] Parker L and Raval A 1999 Phys. Rev. D 60063512

[10] Caldwell R R, Komp W, Parker L and Vanzella D A T 2006 Phys. Rev. D 73023513

[11] Cohen A G, Kaplan D B and Nelson A E 1999 Phys. Rev. Lett. 824971

[12] Padmanabhan T 2005 Class. Quant. Grav. 22 L107

[13] Padmanabhan T and Singh T P 1987 Class. Quant. Grav. 41397

[14] Bombelli L, Koul R K, Lee J-H and Sorkin R D 1986 Phys.Rev. D 34373

[15] Sredinski M 1993 Phys. Rev. Lett. 71666

[16] Brustein R, Eichler D, Foffa S and Oaknin D H 2002 Phys. Rev. D 65105013

[17] Gurzadyan V G and Xue S-S 2003 Mod. Phys. Lett. A 18561

[18] Yarom A and Brustein R 2005 Nucl. Phys. B 709391

[19] Elizalde E, Nojiri S, Odintsov S D and Wang P 2005 Phys. Rev. D 71103504

[20] Zel'dovich Y B 1967 Pis'ma Zh. Theor. Fiz. 61050 [JETP Lett. 6 316]

[21] Cherkas S L, Kalashnikov V L Preprint gr-qc/0604020

[22] Cherkas S L, Kalashnikov V L Preprint astro-ph/0611795

[23] Hartle J B, Hawking S W 1983 Phys. Rev. D 282960

[24] Cherkas S L and Kalashnikov V L 2006 Grav. Cosmol. 12126

[25] Parker L 1971 Phys. Rev. D 3346

[26] Greene P B and Kofman L 2000 Phys. Rev. D 62123516

[27] Peloso M and Sorbo L 2000 JHEP 0005016

[28] Maamache M and Lakehal H 2004 Europhys. Lett. 67695

[29] Zhang Z-G 2006 Phys. Scr. 74218

[30] Berestetskii V B, Lifshitz E M and Pitaevskii L P 1982 Quantum electrodynamics (Oxford, Pergamon Press)

[31] Cherkas S L 1994 Proc. Acad. Sci. Belarus, ser. Fiz.-Mat., 270 [in Russian]

[32] Prokopec T and Woodard R P 2004 Am.J.Phys. 7260

[33] Zel'dovich Ya B and Starobinsky A A 1972 Zh. Eksp. Teor. Fiz. 612161 [JETP 34 (1972) 1159]

[34] Lifshits E M and Khalatnikov I M 1963 Usp. Fiz. Nauk, 80391 [Sov. Phys. Usp. 6 (1964) 496]

[35] Landau L D and Lifshitz E M 2000 The Classical Theory of Fields (Oxford: ButterworthHeinemann)

[36] Commins E D and Buksbaum P H 1983 Weak Interactions of leptons and quarks (Cambrige, University Press) 